\title{
Hilmar Klinkott, Norbert Kramer (Hgg.), Zwischen Assur und Athen - Altorientalisches in den Historien Herodots, (SpielRäume - 9), Franz Steiner Verlag, Stuttgart 2017, S. 243, ISBN 978-3515117432
}

Der hier zu besprechende Band enthält neun Beiträge, die als Referate 2015 an der Ruprechts-Karl-Universität Heidelberg gehalten wurden und die die altorientalischen Elemente in den Historien Herodots thematisieren. Das Hauptanliegen des Sammelbandes sei ,durch eine Art interdisziplinärer Stellenkommentierung zu neuen Blickwinkeln und Deutungen anzuregen“" (S. 8).

Den Band öffnet ein Beitrag von R. Rollinger (Altorientalisches bei Herodot: das wiehernde Pferd des Dareios I., S. 13-42), in dem am Beispiel der berühmten Geschichte (3.84-89) über die Thronbesteigung des Dareios und eine damit verbundene List die Frage behandelt wurde, wie Herodot sich Altorientalischem zunutze machte und in sein Werk einband. ${ }^{1}$ Zuerst macht Rollinger auf die Paralellen aufmerksam, die zwischen dem von Herodot geschilderten Denkmal und einem Relief des urartäischen Königs Rusa, der in einer Inschrift Sargons II. beschreiben wurde, wahrzunehmen sind. Rollinger stellt fest, dass solche Denkmäler wie der, den Dareios in der herodotischen Darstellung errichten ließ, seien zwar im Perserreich nicht zu finden gewesen, aber man solle davon ausgehen, dass „Herodot dieser Tradition in der einen oder anderen Form begegnete und sie sich aneignete, um sie für seine Zwecke zu verändern und in seine Geschichte zu integrieren.“"2 Im zweiten Teil des Artikels (S. 28-42) wurden die einzelnen Elemente, die in der Geschichte über der Oibares' List vorkommen, untersucht, und zwar: das Pferd (ó ï $\pi \pi \circ \varsigma)$; das

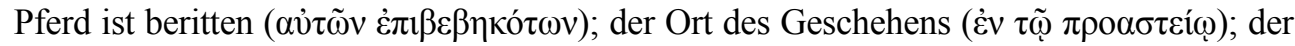

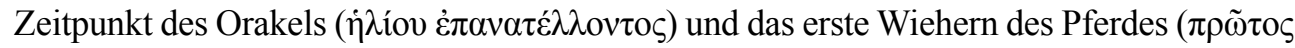
$\varphi \theta \dot{\varepsilon} \gamma \xi \eta \tau \alpha \imath) .{ }^{3}$ Aus der Analyse dieser Bestandteile resultiert, dass ,wir nicht mit einer bloßen Kopie einer älteren altorientalischen Darstellung zu tun haben, sondern mit einer Erzählung, die geschickt in den herodoteischen Gesamtkontext eingefügt ist“" (S. 41).

Eine äußerst spannende und überzeugende Studie bildet der Beitrag von Anthony Ellis (Perser, Meder oder Barbaren? Herodotos Gebrauch der Persernamen und - sitten: zwischen griechischer Literatur und persischer Ethnographie, S. 45-59), der die Frage analysiert, wie Herodot sich den Persernamen und Persersitten verwendete. Von größter Bedeutung ist meines Erachtens die Feststellung, dass die Bedeutung der Ethnonyme „Perser“ und „Meder“ völlig mit dem Kontext der Erzählung verküpft ist: berichtet Herodot über die Auseinandersetztung zwischen den Griechen und den Persern,

${ }^{1}$ Vgl. auch die englische Fassung: R. Rollinger (2018), Herodotus and the Transformation of Ancient Near Eastern Motifs Darius I, Oebares, and the Neighing Horse, in: T. Harrison, E. Irwin (Hgg.), Interpreting Herodotus, Oxford: 125-149.

${ }^{2}$ S. $16-28$.

${ }^{3}$ Hdt 3.84 . 
sind die beiden oben genannten Begriffe eher als Synonyme zu sehen, wohingegen sie eine exakte Bedeutung gewinnen, die auf konkrete Völker hinweist, wenn der Historiker über die Ethnographie zu sprechen kommt. Diese Bemerkung kann eine gute Anregung für eine weitere Untersuchung der von Herodot gebrauchten Begriffe bilden, nicht nur in Bezug auf die Ethno- und Toponymen, sondern auch auf z. B. das Herrschaftvokabular

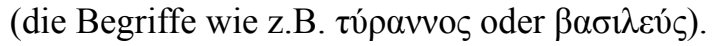

In seinem Artikel setzt sich H. Klinkott (Xerxes und der Kopf des Leonidas. Handlungszwänge und Rollenverständnis eines persischen Großkönigs, S. 61-81) mit der Passage über die Leichenschändung des Leonidas durch Xerxes auseinander (Hdt. 7.238). Der Großkönig ließ - nachdem Leonidas in der Schlacht bei den Thermopylen gefallen war - den Leichnam des Spartaners enthaupten und den Kopf an das Kreuz schlagen oder pfählen $(\dot{\alpha} v \alpha \sigma \tau \alpha v \rho \tilde{\sigma} \sigma \alpha \imath) .{ }^{4}$ Es soll nicht daran gezweifelt werden, dass diese Geschichte eine literarische Ebene hat, die die Grausamkeit des Xerxes hervorhebt. ${ }^{5}$ H. Klinkott weist aber in seinem Text auf eine weitere Dimension hin, und zwar die historische, die die altorientalischen Quellen zutage fördern. Beim Köpfen und Pfälen habe sich nämlich nicht um die Grausamkeit gehandelt, sondern um ,eine konkrete Strafmaßnahme des Königs für diejenigen, die sich gegen die Herrschaft erhoben hatten“ (S. 68). Betrachtet man die Erzählung über die Enthauptung des toten Leonidas aus dieser Perspektive, ließe sich sie auf die folgende Weise interpretieren: durch diese extreme Strafe habe Xerxes seine Autorität betont und ,sich an die königlichen Kritiker im Heer wie auch in den Hofparteien" gewandt (S. 81).

Norbert Kramer (Herkunft, Transformation und Funktion orientalischer Kriegsmotive bei Herodot, S. 83-104) konzentriert sich auf fünf Kriegsmotive, die in den Historien Herodots vorkomen, und zwar Poliorketik, Flucht, Deportation, Inszenierung des Aufmarschs des Königs und Grausamkeit. Der Autor nahm sich als Ziel vor, Herkunft und Funktion dieser Motive zu unteruschen und zu analysieren, auf welche Weise Herodot sie umformfte.

Im darauffolgenden Artikel nimmt Julia Lougovaya-Ast (Das Reiterrelief des Dareios und Herodotos Umgang mit Inschriften, S. 105-121), das Reiterreilef des Dareios - das von R. Rollinger am Anfang des Bandes behandelt wurde - unter die Lupe. Die Autorin plädiert dafür, dass das von Herodot beschriebene Monument in Wirklichkeit existiert habe. Von daher setzt sie sich zum Ziel, ,,den Aufstellungsort des Reliefs und der Inschrift als einer epigraphischen Evidenz innerhalb der Historien und in der Geschichte um Dareios' Thronbesteigung zu untersuchen“" (S. 106). Daraufhin bespricht sie die wissenschaftliche Ansätze, die die Herodots Umgang mit den Inschriften angehen (z.B. der berühmte Beitrag von S. West). ${ }^{6}$ Die Verfasserin macht auf die Diskrepanz zwischen der von Herodot dargestellten Inschrift und der weiteren Erzählung über die Machtübernahme des Dareios aufmerksam: die Inschrift erwähnt lediglich Dareios, wohingegen mehrere Personen in der Erzählung handeln (S. 116-121). Aus dieser Bemerkung zieht die Autorin die Schlussfolgerung, dass man nicht durch Moral, Beachtung der Gesetze zu Macht komme, sondern durch u.a. Skrupellosigkeit und Schlauheit (S. 120-121).

${ }^{4}$ Vgl. J.E. Powell, A Lexicon to Herodotus, Cambridge 1938, s.v. àv $\alpha \sigma \tau \alpha v \rho \tilde{\omega}$.

5 Vgl. z.B. R. Rollinger, Extreme Gewalt und Strafgericht. Ktesias und Herodot als Zeugnisse für den Achaimenidenhof, in: B. Jacobs, R. Rollinger (Hgg.), Der Achämenidenhof, Wiesbaden 2010, 559-666.

${ }^{6}$ S. West, Herodotus' Epigraphical Interests, $C Q 35,1985$, S. 278-305. 
Im nächsten Beitrag untersucht D. Möhlmann (Der Schiffseinsatz bei der AraxesÜberquerung Kyros ' II. - eine Inszenierung persicher Macht?, S. 123-143) den letzten Satz aus dem Kapitel 1.205, in dem von der Überquerung des Flusses Araxes durch Kyros II. während des Krieges gegen die Massageten die Rede ist. Herodot berichtet: ,[Kyros - J.K.] führte sein Heer zum Araxes und fügte Brücken auf dem Fluss zusammen, das Heer hinüberzubringen, und errichtete Türme auf Schiffen, die zuerst den Fluss überqueren sollten (Übersetzung von W. Marg). ${ }^{\text {"7 }}$ Der Artikel von D. Möhlmann besteht aus zwei Hauptteilen. Im ersten Teil wurden die technischen und militärischen Aspekten analysiert, wobei der zweite Teil der Frage gewidmet wurde, ,inwieweit die Flussüberquerung Kyros' II. wie auch der Einsatz der Turm-Schiffe icht auch ein Akt der Inszenierung waren und inwieweit sie auf eine altorientalische Tradition zurückgreifen konnten“ (S. 123-124). In Bezug auf die technischen Fragen kommt der Autor zu dem Ergebnis, dass die von Herodot erwähnten Brücken eine Art von Schiffbrücken gewesen seien. Die Türme seien eher auf den Schiffen errichtet worden, die den Araxes entlang gefahren seien, aber nicht auf den Schiffsbrücken. Die Schiffen und Türme hätten nicht nur dem militärischen, sondern auch dem psychologischen Zweck gedient und zwar der Abschreckung der Feinde. D. Möhlman zeigte darüber hinaus überzeugend, dass die Inszenierungen von Flussüberquerungen im Alten Orient präsent waren, was das Beispiel Assurbanipals II. bestätigt. Kyros II. muss dementsprechend auf eine existierende Tradition zurückgegriffen haben, mithilfe derer er ,seine Führungsqualitäten und den Herrsaftsanspruch Persien“" auszudrücken vermochte (S. 143-144).

Der nächste Text von M. Schuol (Die gepfählten Reiter: Herodotos Skythen-Bild zwischen Realität und Fiktion, S. 145-162) wird der Skythische-Logos thematisiert. Das Hauptanliegen dieser Untersuchung sei ,die Frage nach der Glaubwürdigkeit, der Historitizät und der archäologischen Nachweisbarkeit von Herodots Skythen-Darstellung, "8 wobei die soziale Ausdifferenzierung der Skythen im Fokus der Studie steht (S. 146-147). Die Autorin kommt zu dem Ergebniss, dass die historische Qualität der herodoteischen Skythen-Logos hoch sei, unabhängig davon, dass der Historiker ,,in der Widergabe des ihm zugetragenen Wissens selektiv verfährt, mitunter Lügensgeschichten aufsitzt oder Erzähltes und entsprechend der kompositorischen Gestaltung seines Werkes in abgeänderter Form widergibt“" (S. 161-162). Die zwei letzten Beiträge im Sammelband befassen sich mit der Frage von der achaimenidische Königsideologie in der herodoteischen Erzählung über Xerxes (A. Schwab, Achaimenidische Königsideologie in Herodots Erzählung über Xerxes, Hdt. 7,8-11, S. 163-195) und mit der herodoteischen Darstellung der Magoi (K. Trampedach, Die Priester der Despoten. Herodotos persische Magoi, S. 197-218).

Zusammenfassend kann man feststellen, dass der vorliegende Band eine wichtige und anregende Studie für die Herodot-Forschung ausmacht, die hoffentlich zu einer weiteren Disskusion über das Altorientalische bei Herodot führen wird.

\section{Jakub Kuciak (Jagellonen-Universität zu Krakau)}

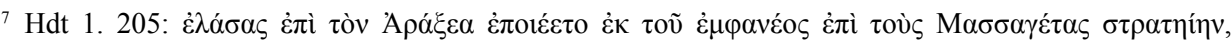

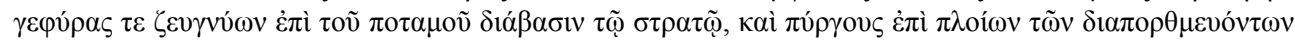

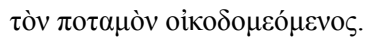

${ }^{8}$ Vgl. auch z.B. F. Hartog, The Mirror of Herodotus - The Representation of the Other in the Writing of History, Berkeley-Los Angeles-London 1988. 